\title{
The Effect of Smoking on Progression From Ductal Carcinoma in Situ to Invasive Ductal Carcinoma: A Retrospective Study
}

\section{Koji Takada}

Department of Breast and Endocrine Surgery, Osaka City University Graduate School of Medicine, 1-4-3 Asahi-machi, Abeno-ku, Osaka 545-8585, Japan

Shinichiro Kashiwagi ( $\square$ spqv9ke9@view.ocn.ne.jp)

Osaka City University Graduate School of Medicine https://orcid.org/0000-0002-0460-9599

\section{Yuka Asano}

Department of Breast and Endocrine Surgery, Osaka City University Graduate School of Medicine, 1-4-3 Asahi-machi, Abeno-ku, Osaka 545-8585, Japan

\section{Wataru Goto}

Department of Breast and Endocrine Surgery, Osaka City University Graduate School of Medicine, 1-4-3 Asahi-machi, Abeno-ku, Osaka 545-8585, Japan

Rika Kouhashi

Department of Breast and Endocrine Surgery, Osaka City University Graduate School of Medicine, 1-4-3 Asahi-machi, Abeno-ku, Osaka 545-8585, Japan

\section{Akimichi Yabumoto}

Department of Breast and Endocrine Surgery, Osaka City University Graduate School of Medicine, 1-4-3 Asahi-machi, Abeno-ku, Osaka 545-8585, Japan

\section{Tamami Morisaki}

Department of Breast and Endocrine Surgery, Osaka City University Graduate School of Medicine, 1-4-3 Asahi-machi, Abeno-ku, Osaka 545-8585, Japan

Masatsune Shibutani

Department of Gastrointestinal Surgery, Osaka City University Graduate School of Medicine, 1-4-3 Asahi-machi, Abeno-ku, Osaka 545-8585, Japan

Tsutomu Takashima

Department of Breast and Endocrine Surgery, Osaka City University Graduate School of Medicine, 1-4-3 Asahi-machi, Abeno-ku, Osaka 545-8585,

Japan

\section{Hisakazu Fujita}

Department of Scientific and Linguistic Fundamentals of Nursing, Osaka City University Graduate School of Nursing, 1-5-17 Asahi-machi, Abeno-ku, Osaka 545-0051, Japan

\section{Kosei Hirakawa}

Department of Breast and Endocrine Surgery, Osaka City University Graduate School of Medicine, 1-4-3 Asahi-machi, Abeno-ku, Osaka 545-8585, Japan. Department of Gastrointestinal Surgery, Osaka City University Graduate School of Medicine, 1-4-3 Asahi-machi,

\section{Masaichi Ohira}

Department of Breast and Endocrine Surgery, Osaka City University Graduate School of Medicine, 1-4-3 Asahi-machi, Abeno-ku, Osaka 545-8585,

Japan. Department of Gastrointestinal Surgery, Osaka City University Graduate School of Medicine, 1-4-3 Asahi-machi,

\section{Research}

Keywords: breast cancer,smoking,ductal carcinoma in situ,invasive ductal carcinoma,biopsy

Posted Date: June 25th, 2020

DOI: https://doi.org/10.21203/rs.3.rs-37789/v1

License: (c) (i) This work is licensed under a Creative Commons Attribution 4.0 International License. Read Full License 


\section{Abstract}

Background.If ductal carcinoma in situ (DCIS) is diagnosed by histological examination such as core needle biopsy or vacuum-assisted biopsy (VAB), invasion is often found by removing the entire tumor and performing pathological examination. According to a meta-analysis, the rate of invasion found by postoperative pathological examination is about $25 \%$. Smoking is a risk factor for carcinogenesis in various carcinomas, including breast cancer. We examined the correlation between the risk of invasion found by postoperative pathology and smoking history in patients who were diagnosed with DCIS by preoperative biopsy.

Methods.In this study, we examined 128 patients who were diagnosed with DCIS by preoperative biopsy. Before the biopsy, all patients were asked about the duration of smoking, number of cigarettes smoked per day, and whether they were currently smoking. The data were used to calculate each patient's tobacco exposure based on pack-years. The statistical analyses included the Pearson's chi-square test and logistic analysis. Multivariate analysis was performed on the risk factors for invasion diagnosed by postoperative pathological examination in all cases diagnosed with DCIS by preoperative biopsy.

Results. Of all the patients included in this study, 107 (83.5\%)neversmoked, which represented the majority, while8 patients (6.3\%) smoked at diagnosis with DCIS, and $13(10.2 \%)$ had quit smokingbefore diagnosis.Tobacco exposure was 10 or less pack-years for 11 patients (8.6\%) and more than 10 pack-years for 10 patients $(7.8 \%)$. Number of pack-years was not an independent factor $(\mathrm{p}=0.349$, odds ratio [OR] 0.329$)$, but current smoker status $(p=0.006, O R=$ not calculable) was an independent factor with $\operatorname{VAB}(p=0.018, O R=0.327)$.

Conclusions. This study suggests that tobacco components may have an influence on the progression from DCIS to invasive ductal carcinoma.

\section{Background}

If ductal carcinoma in situ (DCIS) is diagnosed by histological examination such as core needle biopsy (CNB) or vacuum-assisted biopsy (VAB), invasion is often found by removing the entire tumor and performing pathological examination. According to a meta-analysis, the ratio of invasive ductal carcinoma (IDC) found by postoperative pathological examination is $25.9 \%(18.6-37.2 \%)$ [1]. Various underestimated risk factors for invasion have been reported, including the grade of DCIS [1]. The natural history of the progression from DCIS to IDC is unknown $[9,10]$. However, it is generally believed that the carcinogenesis process for breast cancer progresses from normal breast tissue to atypical ductal hyperplasia, then to DCIS, and lastly to invasive breast cancer [11-13].

Smoking is a risk factor for carcinogenesis in various carcinomas and breast cancer is no exception [2]. Several studies in vivo and in vitro have shown that tobacco smoke components increase breast cancer cell proliferation and cause malignant transformation [3-5]. In reports investigating whether smoking is a risk factor for DCIS, no correlation was found in a large-scale cohort or case-control studies, while another case-control study showed an inverse correlation between smoking and DCIS [6-8].

There has been no report of smoking as a risk factor to underestimate DCIS by biopsy. However, there are reports about tobacco components reaching the mammary gland tissue through the blood, causing DNA damage $[14,15]$. We hypothesized that DCIS may also be affected by smoking components, causing increased malignancy. Based on this hypothesis, we examined the correlation between the risk of invasion found by postoperative pathology and smoking history in patients who were diagnosed with DCIS by preoperative biopsy.

\section{Methods}

\section{Patients' background}

In this study, we examined 128 patients who were diagnosed with DCIS by preoperative biopsy from August 2007 to January 2018 at the Osaka City University Hospital. Before the biopsy, all patients were asked about the duration of smoking, the number of cigarettes smoked per day, and whether they were currently smoking. Based on their smoking status, the patients were classified into never-smokers, current-smokers, and former-smokers. The total number of smokers was the sum of current-smokers and former-smokers. The data were used to calculate each patient's tobacco exposure based on pack-years [16] (Additional file 1: Table S1). Then, all patients were diagnosed with DCIS pathologically by CNB or VAB. The biopsy tissue was used to assess the grade of DCIS based on the World Health Organization classification [17], comedo necrosis, intraluminal calcification, and interstitial inflammation (as previously reported $[18,19]$ ). Immunohistochemical staining was performed in the biopsy tissue to evaluate the expression of the estrogen receptor (ER), progesterone receptor (PgR), human epidermal growth factor receptor 2 (HER2), and Ki67 in cancer cells. Tumor size was measured by ultrasonography, computed tomography (CT), and magnetic resonance imaging. Cases suspected of invasion or metastasis by these imaging techniques were excluded. About half of all patients did not undergo mammography. All patients underwent mastectomy or breast-conserving surgery after being diagnosed with DCIS.

\section{Statistical analysis}

The JMP software package version 15 (SAS, Tokyo, Japan) was used for all statistical analysis. The relationship between each factor was examined by the Pearson's chi-square test. Logistic analysis was used for calculating the odds ratio (OR) and $95 \%$ confidence interval. The multivariate logistic 
regression model was used for multivariate analysis. A p-value lower than 0.05 was defined as significant.

\section{Ethics statement}

This study was conducted at the Osaka City University Graduate School of Medicine (Osaka, Japan). The study protocol involved a retrospectively written research, pathological evaluation, and statistical analysis plan. The study complied with the provisions of the Declaration of Helsinki, and all patients provided written informed consent for their treatment and data collection. The study's retrospective protocol was approved by the ethics committee of Osaka City University (approval number \#926).

\section{Results}

\section{Clinicopathological features}

The clinicopathological features of 128 patients diagnosed with DCIS by preoperative biopsy are listed in Table 1 . The median age was 51 years (range, 30-78 years), and 17 patients (13.2\%) were under 40 years old. Eighty-three patients (64.8\%) had some symptoms, and the tumor was palpable at medical consultation in ninety-three patients (72.7\%). Forty-five patients (35.2\%) were asymptomatic and were found by routine screening for breast cancer or CT examination for other diseases. The median tumor diameter was $17.7 \mathrm{~mm}$ (range, 3.0-50.0 mm). For the biopsy method, 73 patients (57.0\%) were diagnosed by CNB, which was more than that by VAB. The expression of ER and PgR were positive in 104 (81.3\%) and 87 (68.0\%) DCIS cases, respectively. Regarding HER2, the score was $2+$ in 22 patients (17.2\%) and $3+$ in 19 patients (14.8\%). The expression of Ki67 was higher than $14 \%$ in 27 patients (21.1\%). Regarding the grade of DCIS, 53 patients (41.4\%) had low-grade, 51 (39.8\%) had intermediate-grade, and $24(18.8 \%)$ had high-grade DCIS. In the biopsy specimens, comedo necrosis was found in 68 patients (53.1\%), and intraductal calcification was found in 21 patients (16.4\%). Lymphoid infiltrate was classified into four stages: Stage1, Stage2, Stage3, and Stage4; it was evaluated as moderate or severe in 39 patients (30.4\%). IDC was found by postoperative pathological examination in 50 patients (39.1\%). 
Table 1

Clinicopathological features of 128 cases diagnosed with DCIS by preoperative biopsy

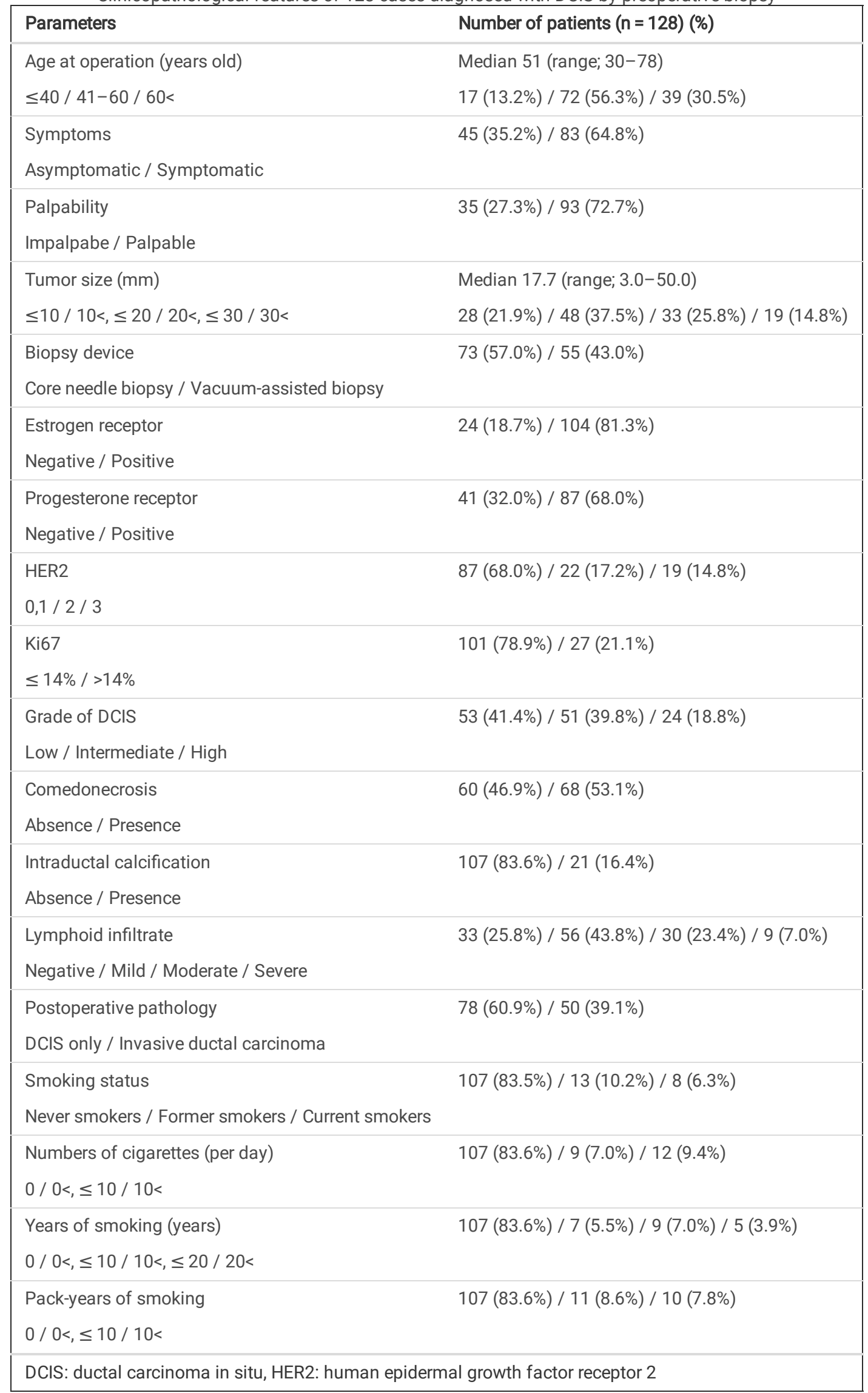

Regarding smoking, the majority of patients were never-smokers (107 patients, 83.5\%). Eight patients (6.3\%) smoked at diagnosis of DCIS (currentsmokers), and thirteen patients (10.2\%) had quit smoking before diagnosis (former-smokers). Twelve patients (9.4\%) smoked more than ten cigarettes per day, which were more than half of the total number of smokers. Regarding the period of smoking, 7 patients (5.5\%) smoked for 
10 years or less, 9 patients (7.0\%) smoked for 11 to 20 years, and 5 patients (3.9\%) smoked for more than 20 years. Tobacco exposure was 10 or less pack-years for 11 patients (8.6\%) and more than 10 pack-years for 10 patients (7.8\%).

\section{Risk factors for finding invasion in postoperative pathological examination (univariate analysis)}

Univariate analysis was performed on clinicopathologic features in which invasion was found in postoperative pathology, and the results are shown in Table 2. IDC tended to be found postoperatively in patients who had symptoms at diagnosis $(p=0.082,0 R=1.980)$ or whose tumor was palpable $(p=0.058, O R=2.278)$. The $O R$ and tumor size were positively correlated, and tumors larger than 30 mm were significantly more invasive than those smaller than $10 \mathrm{~mm}(p=0.015, O R=4.518)$. DCIS diagnosed by VAB was significantly more invasive than $D C I S$ diagnosed by $C N B(p=0.018, O R=$ 0.407). Invasion was found more frequently in postoperative pathology in ER negative DCIS than in ER positive DCIS ( $p=0.009,0 R=0.304)$. The relation with PgR was similar but not significant $(p=0.053, O R=0.476)$. Regarding HER2, patients with a score of 3 had more invasion than did patients with scores of 0 or $1(p=0.027, O R=3.097)$. DCIS with high Ki67 had a significantly higher risk of finding IDC than did DCIS with low Ki67 ( $p$ $=0.016, \mathrm{OR}=0.304)$. High-grade DCIS was significantly more likely to have IDC in postoperative pathological diagnosis than did low-grade DCIS $(p=$ $0.007, \mathrm{OR}=3.889$ ). With respect to lymphoid infiltrate, the risk of finding invasion increased as the density of lymphocytes surrounding DCIS increased. As a result, compared with negative-lymphoid infiltrate, invasion was found to be significant in DCIS that had moderate $(p=0.004,0 R=$ $4.606)$ or severe $(p=0.029, \mathrm{OR}=5.333)$ infiltrates. 
Table 2

Univariate analysis with postoperative pathology

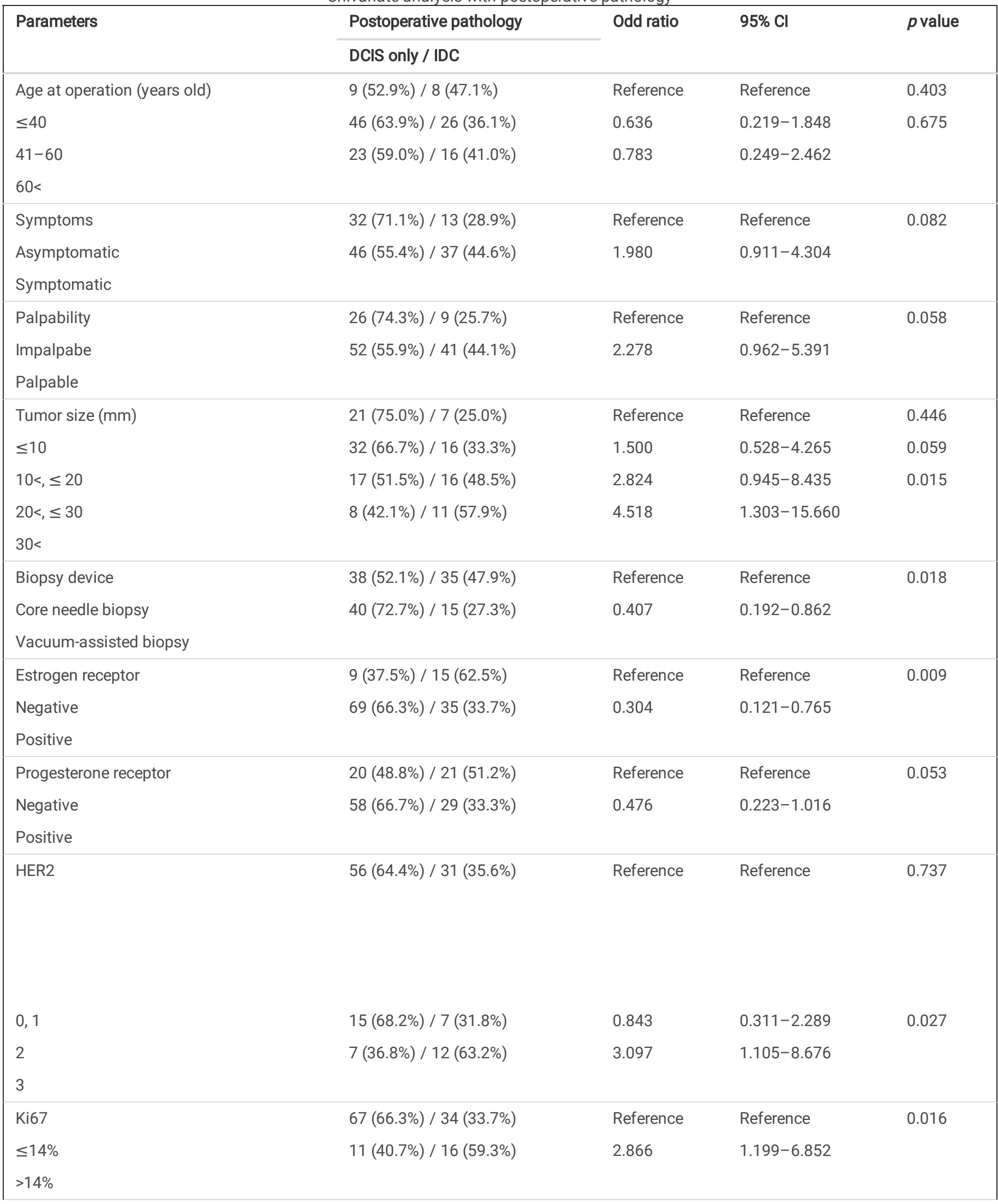




\begin{tabular}{|c|c|c|c|c|}
\hline \multirow[t]{2}{*}{ Parameters } & Postoperative pathology & \multirow[t]{2}{*}{ Odd ratio } & \multirow[t]{2}{*}{$95 \% \mathrm{Cl}$} & \multirow[t]{2}{*}{$p$ value } \\
\hline & DCIS only / IDC & & & \\
\hline Grade of DCIS & 35 (66.0\%) / 18 (34.0\%) & Reference & Reference & 0.778 \\
\hline Low & 35 (68.6\%) / 16 (31.4\%) & 0.889 & $0.391-2.019$ & 0.007 \\
\hline Intermediate & 8 (33.3\%) / 16 (66.7\%) & 3.889 & $1.400-10.801$ & \\
\hline \multicolumn{5}{|l|}{ High } \\
\hline Comedonecrosis & 41 (68.3\%) / 19 (31.7\%) & Reference & Reference & 0.107 \\
\hline Absence & 37 (54.4\%) / 31 (45.6\%) & 1.808 & $0.877-3.728$ & \\
\hline \multicolumn{5}{|l|}{ Presence } \\
\hline Intraductal calcification & 68 (63.6\%) / 39 (36.4\%) & Reference & Reference & 0.171 \\
\hline Absence & 10 (47.6\%) / 11 (52.4\%) & 1.918 & $0.747-4.922$ & \\
\hline \multicolumn{5}{|l|}{ Presence } \\
\hline Lymphoid infiltrate & $24(72.7 \%) / 9(27.3 \%)$ & Reference & Reference & 0.895 \\
\hline Negative & 40 (71.4\%) / $16(28.6 \%)$ & 1.067 & $0.408-2.788$ & 0.004 \\
\hline Mild & $11(36.7 \%) / 19$ (63.3\%) & 4.606 & $1.585-13.387$ & 0.029 \\
\hline Moderate & $3(33.3 \%) / 6(66.7 \%)$ & 5.333 & $1.095-25.985$ & \\
\hline \multicolumn{5}{|l|}{ Severe } \\
\hline Smoking status & 63 (58.9\%) / 44 (41.1\%) & Reference & Reference & 0.728 \\
\hline Never smokers & $7(53.8 \%) / 6(46.2 \%)$ & 1.227 & $0.386-3.901$ & 0.021 \\
\hline Former smokers & $8(100.0 \%) / 0(0.0 \%)$ & - & - & \\
\hline \multicolumn{5}{|l|}{ Current smokers } \\
\hline Numbers of cigarettes (per day) & 63 (58.9\%) / 44 (41.1\%) & Reference & Reference & 0.648 \\
\hline 0 & $6(66.7 \%) / 3(33.3 \%)$ & 0.716 & $0.170-3.017$ & 0.279 \\
\hline $0<, \leq 10$ & 9 (75.0\%) / $3(25.0 \%)$ & 0.477 & $0.122-1.863$ & \\
\hline \multicolumn{5}{|l|}{$10<$} \\
\hline Years of smoking & 63 (58.9\%) / 44 (41.1\%) & Reference & Reference & 0.928 \\
\hline 0 & $4(57.1 \%) / 3(42.9 \%)$ & 1.074 & $0.229-5.038$ & 0.266 \\
\hline $0<, \leq 10$ & $7(77.8 \%) / 2(22.2 \%)$ & 0.409 & $0.081-2.063$ & 0.346 \\
\hline $10<, \leq 20$ & $4(80.0 \%) / 1(20.0 \%)$ & 0.358 & $0.039-3.312$ & \\
\hline \multicolumn{5}{|l|}{$20<$} \\
\hline Pack-years of smoking & 63 (58.9\%) / 44 (41.1\%) & Reference & Reference & 0.781 \\
\hline 0 & $6(54.5 \%) / 5(45.5 \%)$ & 1.193 & $0.342-4.155$ & 0.053 \\
\hline $0<, \leq 10$ & $9(90.0 \%) / 1(10.0 \%)$ & 0.159 & $0.019-1.301$ & \\
\hline $10<$ & & & & \\
\hline
\end{tabular}

Regarding smoking, no significant difference was found between never-smokers and smokers. However, no current-smokers were found to have IDC by postoperative pathological examination, which was significantly different from never-smokers $(p=0.021,0 R=$ cannot be calculated). As the number of cigarettes smoked per day and the smoking period increased, the OR decreased, but it was not significant. When examined by pack-years, IDC tended to be harder to find in patients with more than 10 pack-years of tobacco exposure than in never-smokers $(p=0.053,0 R=0.159)$.

\section{Correlations between clinicopathological features and smoking status}

The correlations between smoking status and clinicopathological features showed that the number of smokers was significantly higher in younger patients (under 40 years old) than in middle age or older patients (over 40 years old) $(p<0.001)$ (Table 3). Former-smokers and current-smokers were significantly younger than never-smokers $(p=0.008, p<0.001$ respectively). Current-smokers presented with significantly more symptoms than did 
never-smokers $(p=0.029)$. However, no other clinicopathological features differed based on smoking status. There was no correlation with any clinicopathological features when divided into two groups with a cutoff value of 10 pack-years. 
Table 3

Correlation between smoking status and clinicopathological features

\begin{tabular}{|c|c|c|c|c|c|c|c|c|c|c|c|c|}
\hline \multirow[t]{2}{*}{ Parameters } & \multicolumn{2}{|l|}{ Smoker } & \multirow{2}{*}{$\begin{array}{l}p \\
\text { value }\end{array}$} & \multicolumn{2}{|c|}{ Smoking status } & \multirow{2}{*}{$\begin{array}{l}p \\
\text { value }\end{array}$} & \multicolumn{2}{|c|}{ Smoking status } & \multirow{2}{*}{$p_{\text {value }}^{p}$} & \multicolumn{2}{|c|}{ Pack-years } & \multirow{2}{*}{$\begin{array}{l}p \\
\text { value }\end{array}$} \\
\hline & $\begin{array}{l}\text { Never } \\
\text { smoker } \\
(\mathrm{n}= \\
107)\end{array}$ & $\begin{array}{l}\text { Smoker } \\
(n=21)\end{array}$ & & $\begin{array}{l}\text { Never } \\
\text { smoker } \\
(n= \\
107)\end{array}$ & $\begin{array}{l}\text { Former } \\
\text { smokers } \\
(n=13)\end{array}$ & & $\begin{array}{l}\text { Never } \\
\text { smoker } \\
\text { (n= } \\
107)\end{array}$ & $\begin{array}{l}\text { Current } \\
\text { smokers } \\
(n=8)\end{array}$ & & $\begin{array}{l}\leq 10 \\
(n= \\
118)\end{array}$ & $\begin{array}{l}10< \\
(n=10)\end{array}$ & \\
\hline $\begin{array}{l}\text { Age at operation } \\
\text { (years old) }\end{array}$ & $\begin{array}{l}8 \\
(7.5 \%)\end{array}$ & $\begin{array}{l}9 \\
(42.9 \%)\end{array}$ & $<.001$ & $\begin{array}{l}8 \\
(7.5 \%)\end{array}$ & $\begin{array}{l}4 \\
(30.8 \%)\end{array}$ & 0.008 & $\begin{array}{l}8 \\
(7.5 \%)\end{array}$ & $\begin{array}{l}5 \\
(62.5 \%)\end{array}$ & $<.001$ & $\begin{array}{l}14 \\
(11.9 \%)\end{array}$ & $\begin{array}{l}3 \\
(30.0 \%)\end{array}$ & 0.105 \\
\hline $\begin{array}{l}\leq 40 \\
>40\end{array}$ & $\begin{array}{l}99 \\
(92.5 \%)\end{array}$ & $\begin{array}{l}12 \\
(57.1 \%)\end{array}$ & & $\begin{array}{l}99 \\
(92.5 \%)\end{array}$ & $\begin{array}{l}9 \\
(69.2 \%)\end{array}$ & & $\begin{array}{l}99 \\
(92.5 \%)\end{array}$ & $\begin{array}{l}3 \\
(37.5 \%)\end{array}$ & & $\begin{array}{l}104 \\
(88.1 \%)\end{array}$ & $\begin{array}{l}7 \\
(70.0 \%)\end{array}$ & \\
\hline $\begin{array}{l}\text { Symptoms } \\
\text { Asymptomatic } \\
\text { Symptomatic }\end{array}$ & $\begin{array}{l}41 \\
(38.3 \%) \\
66 \\
(61.7 \%)\end{array}$ & $\begin{array}{l}4 \\
(19.0 \%) \\
17 \\
(81.0 \%)\end{array}$ & 0.091 & $\begin{array}{l}41 \\
(38.3 \%) \\
66 \\
(61.7 \%)\end{array}$ & $\begin{array}{l}4 \\
(30.8 \%) \\
9 \\
(69.2 \%)\end{array}$ & 0.596 & $\begin{array}{l}41 \\
(38.3 \%) \\
66 \\
(61.7 \%)\end{array}$ & $\begin{array}{l}0(0.0 \%) \\
8 \\
(100.0 \%)\end{array}$ & 0.029 & $\begin{array}{l}43 \\
(36.4 \%) \\
75 \\
(63.6 \%)\end{array}$ & $\begin{array}{l}2 \\
(20.0 \%) \\
8 \\
(80.0 \%)\end{array}$ & 0.296 \\
\hline $\begin{array}{l}\text { Palpability } \\
\text { Impalpabe } \\
\text { Palpable }\end{array}$ & $\begin{array}{l}32 \\
(29.9 \%) \\
75 \\
(70.1 \%)\end{array}$ & $\begin{array}{l}3 \\
(14.3 \%) \\
18 \\
(85.7 \%)\end{array}$ & 0.142 & $\begin{array}{l}32 \\
(29.9 \%) \\
75 \\
(70.1 \%)\end{array}$ & $\begin{array}{l}3 \\
(23.1 \%) \\
10 \\
(76.9 \%)\end{array}$ & 0.609 & $\begin{array}{l}32 \\
(29.9 \%) \\
75 \\
(70.1 \%)\end{array}$ & $\begin{array}{l}0(0.0 \%) \\
8 \\
(100.0 \%)\end{array}$ & 0.069 & $\begin{array}{l}32 \\
(27.1 \%) \\
86 \\
(72.9 \%)\end{array}$ & $\begin{array}{l}3 \\
(30.0 \%) \\
7 \\
(70.0 \%)\end{array}$ & 0.844 \\
\hline $\begin{array}{l}\text { Tumor size } \\
(\mathrm{mm})\end{array}$ & $\begin{array}{l}65 \\
(60.7 \%)\end{array}$ & $\begin{array}{l}11 \\
(52.4 \%)\end{array}$ & 0.475 & $\begin{array}{l}65 \\
(60.7 \%)\end{array}$ & $\begin{array}{l}8 \\
(61.5 \%)\end{array}$ & 0.956 & $\begin{array}{l}65 \\
(60.7 \%)\end{array}$ & $\begin{array}{l}3 \\
(37.5 \%)\end{array}$ & 0.197 & $\begin{array}{l}69 \\
(58.5 \%)\end{array}$ & $\begin{array}{l}7 \\
(70.0 \%)\end{array}$ & 0.476 \\
\hline $\begin{array}{l}\leq 20.0 \\
>20.0\end{array}$ & $\begin{array}{l}42 \\
(39.3 \%)\end{array}$ & $\begin{array}{l}10 \\
(47.6 \%)\end{array}$ & & $\begin{array}{l}42 \\
(39.3 \%)\end{array}$ & $\begin{array}{l}5 \\
(38.5 \%)\end{array}$ & & $\begin{array}{l}42 \\
(39.3 \%)\end{array}$ & $\begin{array}{l}5 \\
(62.5 \%)\end{array}$ & & $\begin{array}{l}49 \\
(41.5 \%)\end{array}$ & $\begin{array}{l}3 \\
(30.0 \%)\end{array}$ & \\
\hline $\begin{array}{l}\text { Biopsy device } \\
\text { Core needle } \\
\text { biopsy } \\
\text { Vacuum- } \\
\text { assisted biopsy }\end{array}$ & $\begin{array}{l}63 \\
(58.9 \%) \\
44 \\
(41.1 \%)\end{array}$ & $\begin{array}{l}10 \\
(47.6 \%) \\
11 \\
(52.4 \%)\end{array}$ & 0.341 & $\begin{array}{l}63 \\
(58.9 \%) \\
44 \\
(41.1 \%)\end{array}$ & $\begin{array}{l}8 \\
(61.5 \%) \\
5 \\
(38.5 \%)\end{array}$ & 0.854 & $\begin{array}{l}63 \\
(58.9 \%) \\
44 \\
(41.1 \%)\end{array}$ & $\begin{array}{l}2 \\
(25.0 \%) \\
6 \\
(75.0 \%)\end{array}$ & 0.062 & $\begin{array}{l}69 \\
(58.5 \%) \\
49 \\
(41.5 \%)\end{array}$ & $\begin{array}{l}4 \\
(40.0 \%) \\
6 \\
(60.0 \%)\end{array}$ & 0.257 \\
\hline $\begin{array}{l}\text { Estrogen } \\
\text { receptor }\end{array}$ & $\begin{array}{l}19 \\
(17.8 \%)\end{array}$ & $\begin{array}{l}5 \\
(23.8 \%)\end{array}$ & 0.516 & $\begin{array}{l}19 \\
(17.8 \%)\end{array}$ & $\begin{array}{l}4 \\
(30.8 \%)\end{array}$ & 0.260 & $\begin{array}{l}19 \\
(17.8 \%)\end{array}$ & $\begin{array}{l}1 \\
(12.5 \%)\end{array}$ & 0.705 & $\begin{array}{l}23 \\
(19.5 \%)\end{array}$ & $\begin{array}{l}1 \\
(10.0 \%)\end{array}$ & 0.460 \\
\hline $\begin{array}{l}\text { Negative } \\
\text { Positive }\end{array}$ & $\begin{array}{l}88 \\
(82.2 \%)\end{array}$ & $\begin{array}{l}16 \\
(76.2 \%)\end{array}$ & & $\begin{array}{l}88 \\
(82.2 \%)\end{array}$ & $\begin{array}{l}9 \\
(69.2 \%)\end{array}$ & & $\begin{array}{l}88 \\
(82.2 \%)\end{array}$ & $\begin{array}{l}7 \\
(87.5 \%)\end{array}$ & & $\begin{array}{l}95 \\
(80.5 \%)\end{array}$ & $\begin{array}{l}9 \\
(90.0 \%)\end{array}$ & \\
\hline $\begin{array}{l}\text { Progesterone } \\
\text { receptor }\end{array}$ & $\begin{array}{l}35 \\
(32.7 \%)\end{array}$ & $\begin{array}{l}6 \\
(28.6 \%)\end{array}$ & 0.710 & $\begin{array}{l}35 \\
(32.7 \%)\end{array}$ & $\begin{array}{l}4 \\
(30.8 \%)\end{array}$ & 0.888 & $\begin{array}{l}35 \\
(32.7 \%)\end{array}$ & $\begin{array}{l}2 \\
(25.0 \%)\end{array}$ & 0.652 & $\begin{array}{l}39 \\
(33.1 \%)\end{array}$ & $\begin{array}{l}2 \\
(20.0 \%)\end{array}$ & 0.396 \\
\hline $\begin{array}{l}\text { Negative } \\
\text { Positive }\end{array}$ & $\begin{array}{l}72 \\
(67.3 \%)\end{array}$ & $\begin{array}{l}15 \\
(71.4 \%)\end{array}$ & & $\begin{array}{l}72 \\
(67.3 \%)\end{array}$ & $\begin{array}{l}9 \\
(69.2 \%)\end{array}$ & & $\begin{array}{l}72 \\
(67.3 \%)\end{array}$ & $\begin{array}{l}6 \\
(75.0 \%)\end{array}$ & & $\begin{array}{l}79 \\
(66.9 \%)\end{array}$ & $\begin{array}{l}8 \\
(80.0 \%)\end{array}$ & \\
\hline $\begin{array}{l}\text { HER2 } \\
\leq 2 \\
3\end{array}$ & $\begin{array}{l}91 \\
(85.0 \%) \\
16 \\
(15.0 \%)\end{array}$ & $\begin{array}{l}18 \\
(85.7 \%) \\
3 \\
(14.3 \%)\end{array}$ & 0.937 & $\begin{array}{l}91 \\
(85.0 \%) \\
16 \\
(15.0 \%)\end{array}$ & $\begin{array}{l}10 \\
(76.9 \%) \\
3 \\
(23.1 \%)\end{array}$ & 0.449 & $\begin{array}{l}91 \\
(85.0 \%) \\
16 \\
(15.0 \%)\end{array}$ & $\begin{array}{l}8 \\
(100.0 \%) \\
0(0.0 \%)\end{array}$ & 0.238 & $\begin{array}{l}101 \\
(85.6 \%) \\
17 \\
(14.4 \%)\end{array}$ & $\begin{array}{l}8 \\
(80.0 \%) \\
2 \\
(20.0 \%)\end{array}$ & 0.633 \\
\hline $\begin{array}{l}\text { Ki67 } \\
\leq 14 \% \\
>14 \%\end{array}$ & $\begin{array}{l}86 \\
(80.4 \%) \\
21 \\
(19.6 \%)\end{array}$ & $\begin{array}{l}15 \\
(71.4 \%) \\
6 \\
(28.6 \%)\end{array}$ & 0.358 & $\begin{array}{l}86 \\
(80.4 \%) \\
21 \\
(19.6 \%)\end{array}$ & $\begin{array}{l}10 \\
(76.9 \%) \\
3 \\
(23.1 \%)\end{array}$ & 0.769 & $\begin{array}{l}86 \\
(80.4 \%) \\
21 \\
(19.6 \%)\end{array}$ & $\begin{array}{l}5 \\
(62.5 \%) \\
3 \\
(37.5 \%)\end{array}$ & 0.230 & $\begin{array}{l}93 \\
(78.8 \%) \\
25 \\
(21.2 \%)\end{array}$ & $\begin{array}{l}8 \\
(80.0 \%) \\
2 \\
(20.0 \%)\end{array}$ & 0.930 \\
\hline $\begin{array}{l}\text { Grade of DCIS } \\
\text { Low, } \\
\text { intermediate } \\
\text { High }\end{array}$ & $\begin{array}{l}85 \\
(79.4 \%) \\
22 \\
(20.6 \%)\end{array}$ & $\begin{array}{l}18 \\
(85.7 \%) \\
3 \\
(14.3 \%)\end{array}$ & 0.507 & $\begin{array}{l}85 \\
(79.4 \%) \\
22 \\
(20.6 \%)\end{array}$ & $\begin{array}{l}11 \\
(84.6 \%) \\
2 \\
(15.4 \%)\end{array}$ & 0.660 & $\begin{array}{l}85 \\
(79.4 \%) \\
22 \\
(20.6 \%)\end{array}$ & $\begin{array}{l}7 \\
(87.5 \%) \\
1 \\
(12.5 \%)\end{array}$ & 0.582 & $\begin{array}{l}93 \\
(78.8 \%) \\
25 \\
(21.2 \%)\end{array}$ & $\begin{array}{l}10 \\
(100.0 \%) \\
0(0.0 \%)\end{array}$ & 0.105 \\
\hline
\end{tabular}




\begin{tabular}{|c|c|c|c|c|c|c|c|c|c|c|c|c|}
\hline \multirow[t]{2}{*}{ Parameters } & \multicolumn{2}{|l|}{ Smoker } & \multirow{2}{*}{$\begin{array}{l}p \\
\text { value }\end{array}$} & \multicolumn{2}{|c|}{ Smoking status } & \multirow{2}{*}{$\begin{array}{l}p \\
\text { value }\end{array}$} & \multicolumn{2}{|c|}{ Smoking status } & \multirow{2}{*}{$\begin{array}{l}p \\
\text { value }\end{array}$} & \multicolumn{2}{|c|}{ Pack-years } & \multirow{2}{*}{$\begin{array}{l}p \\
\text { value }\end{array}$} \\
\hline & $\begin{array}{l}\text { Never } \\
\text { smoker } \\
(n= \\
107)\end{array}$ & $\begin{array}{l}\text { Smoker } \\
(n=21)\end{array}$ & & $\begin{array}{l}\text { Never } \\
\text { smoker } \\
(n= \\
107)\end{array}$ & $\begin{array}{l}\text { Former } \\
\text { smokers } \\
(n=13)\end{array}$ & & $\begin{array}{l}\text { Never } \\
\text { smoker } \\
(n= \\
107)\end{array}$ & $\begin{array}{l}\text { Current } \\
\text { smokers } \\
(n=8)\end{array}$ & & $\begin{array}{l}\leq 10 \\
(n= \\
118)\end{array}$ & $\begin{array}{l}10< \\
(n=10)\end{array}$ & \\
\hline \multirow{2}{*}{$\begin{array}{l}\text { Comedonecrosis } \\
\text { Absence } \\
\text { Presence }\end{array}$} & $\begin{array}{l}49 \\
(45.8 \%)\end{array}$ & $\begin{array}{l}11 \\
(52.4 \%)\end{array}$ & 0.580 & $\begin{array}{l}49 \\
(45.8 \%)\end{array}$ & $\begin{array}{l}7 \\
(53.8 \%)\end{array}$ & 0.583 & $\begin{array}{l}49 \\
(45.8 \%)\end{array}$ & $\begin{array}{l}4 \\
(50.0 \%)\end{array}$ & 0.818 & $\begin{array}{l}55 \\
(46.6 \%)\end{array}$ & $\begin{array}{l}5 \\
(50.0 \%)\end{array}$ & \multirow[t]{2}{*}{0.837} \\
\hline & $\begin{array}{l}58 \\
(54.2 \%)\end{array}$ & $\begin{array}{l}10 \\
(47.6 \%)\end{array}$ & & $\begin{array}{l}58 \\
(54.2 \%)\end{array}$ & $\begin{array}{l}6 \\
(46.2 \%)\end{array}$ & & $\begin{array}{l}58 \\
(54.2 \%)\end{array}$ & $\begin{array}{l}4 \\
(50.0 \%)\end{array}$ & & $\begin{array}{l}63 \\
(53.4 \%)\end{array}$ & $\begin{array}{l}5 \\
(50.0 \%)\end{array}$ & \\
\hline $\begin{array}{l}\text { Intraductal } \\
\text { calcification }\end{array}$ & $\begin{array}{l}89 \\
(83.2 \%)\end{array}$ & $\begin{array}{l}18 \\
(85.7 \%)\end{array}$ & 0.774 & $\begin{array}{l}89 \\
(83.2 \%)\end{array}$ & $\begin{array}{l}11 \\
(84.6 \%)\end{array}$ & 0.895 & $\begin{array}{l}89 \\
(83.2 \%)\end{array}$ & $\begin{array}{l}7 \\
(87.5 \%)\end{array}$ & 0.751 & $\begin{array}{l}99 \\
(83.9 \%)\end{array}$ & $\begin{array}{l}8 \\
(80.0 \%)\end{array}$ & 0.749 \\
\hline $\begin{array}{l}\text { Absence } \\
\text { Presence }\end{array}$ & $\begin{array}{l}18 \\
(16.8 \%)\end{array}$ & $\begin{array}{l}3 \\
(14.3 \%)\end{array}$ & & $\begin{array}{l}18 \\
(16.8 \%)\end{array}$ & $\begin{array}{l}2 \\
(15.4 \%)\end{array}$ & & $\begin{array}{l}18 \\
(16.8 \%)\end{array}$ & $\begin{array}{l}1 \\
(12.5 \%)\end{array}$ & & $\begin{array}{l}19 \\
(16.1 \%)\end{array}$ & $\begin{array}{l}2 \\
(20.0 \%)\end{array}$ & \\
\hline $\begin{array}{l}\text { Lymphoid } \\
\text { infiltrate }\end{array}$ & $\begin{array}{l}73 \\
(68.2 \%)\end{array}$ & $\begin{array}{l}16 \\
(76.2 \%)\end{array}$ & 0.468 & $\begin{array}{l}73 \\
(68.2 \%)\end{array}$ & $\begin{array}{l}9 \\
(69.2 \%)\end{array}$ & 0.941 & $\begin{array}{l}73 \\
(68.2 \%)\end{array}$ & $\begin{array}{l}7 \\
(87.5 \%)\end{array}$ & 0.253 & $\begin{array}{l}81 \\
(68.6 \%)\end{array}$ & $\begin{array}{l}8 \\
(80.0 \%)\end{array}$ & 0.454 \\
\hline $\begin{array}{l}\text { Negative, mild } \\
\text { Moderate, } \\
\text { severe }\end{array}$ & $\begin{array}{l}34 \\
(31.8 \%)\end{array}$ & $\begin{array}{l}5 \\
(23.8 \%)\end{array}$ & & $\begin{array}{l}34 \\
(31.8 \%)\end{array}$ & $\begin{array}{l}4 \\
(30.8 \%)\end{array}$ & & $\begin{array}{l}34 \\
(31.8 \%)\end{array}$ & $\begin{array}{l}1 \\
(12.5 \%)\end{array}$ & & $\begin{array}{l}37 \\
(31.4 \%)\end{array}$ & $\begin{array}{l}2 \\
(20.0 \%)\end{array}$ & \\
\hline $\begin{array}{l}\text { Postoperative } \\
\text { pathology }\end{array}$ & $\begin{array}{l}63 \\
(58.9 \%)\end{array}$ & $\begin{array}{l}15 \\
(71.4 \%)\end{array}$ & 0.281 & $\begin{array}{l}63 \\
(58.9 \%)\end{array}$ & $\begin{array}{l}7 \\
(53.8 \%)\end{array}$ & 0.728 & $\begin{array}{l}63 \\
(58.9 \%)\end{array}$ & $\begin{array}{l}8 \\
(100.0 \%)\end{array}$ & 0.021 & $\begin{array}{l}69 \\
(58.5 \%)\end{array}$ & $\begin{array}{l}9 \\
(90.0 \%)\end{array}$ & 0.050 \\
\hline $\begin{array}{l}\text { DCIS only } \\
\text { Invasive ductal } \\
\text { carcinoma }\end{array}$ & $\begin{array}{l}44 \\
(41.1 \%)\end{array}$ & $\begin{array}{l}6 \\
(28.6 \%)\end{array}$ & & $\begin{array}{l}44 \\
(41.1 \%)\end{array}$ & $\begin{array}{l}6 \\
(46.2 \%)\end{array}$ & & $\begin{array}{l}44 \\
(41.1 \%)\end{array}$ & $0(0.0 \%)$ & & $\begin{array}{l}49 \\
(41.5 \%)\end{array}$ & $\begin{array}{l}1 \\
(10.0 \%)\end{array}$ & \\
\hline
\end{tabular}

Comparing former-smokers and current-smokers, former-smokers smoked significantly fewer cigarettes per day $(p=0.027)$ and had significantly more invasion in postoperative pathology $(p=0.023)$ (Additional file 2: Table S2).

Risk factors for finding invasion in postoperative pathological examination (multivariate analysis)

Multivariate analysis was performed on the risk factors for invasion diagnosed by postoperative pathological examination in all cases diagnosed with DCIS by preoperative biopsy (Table 4$)$. Number of pack-years was not an independent factor $(p=0.349,0 R=0.329)$, but current-smoker status $(p=0.006, O R=$ not calculable $)$ was an independent factor with $\operatorname{VAB}(p=0.018, O R=0.327)$. 
Table 4

Univariate and multivariate analysis with upstaging preoperatively DCIS to invasive cancer.

\begin{tabular}{|c|c|c|c|c|c|c|}
\hline \multirow[b]{2}{*}{ Parameters } & \multicolumn{3}{|c|}{ Univarite analysis } & \multicolumn{3}{|c|}{ Multivarite analysis } \\
\hline & Odd ratio & $95 \% \mathrm{Cl}$ & $p$ value & Odd ratio & $95 \% \mathrm{Cl}$ & $p$ value \\
\hline $\begin{array}{l}\text { Age at operation (years old) } \\
\leq 40 \text { vs }>40\end{array}$ & 0.685 & $0.245-1.911$ & 0.468 & & & \\
\hline $\begin{array}{l}\text { Symptoms } \\
\text { Asymptomatic vs Symptoma }\end{array}$ & 1.980 & $0.911-4.304$ & 0.082 & 2.809 & $0.794-10.717$ & 0.110 \\
\hline $\begin{array}{l}\text { Palpability } \\
\text { Impalpabe vs Palpable }\end{array}$ & 2.278 & $0.962-5.391$ & 0.058 & 0.764 & $0.183-3.124$ & 0.707 \\
\hline $\begin{array}{l}\text { Tumor size }(\mathrm{mm}) \\
\leq 20.0 \text { vs }>20.0\end{array}$ & 2.489 & $1.197-5.173$ & 0.014 & 2.618 & $0.979-7.343$ & 0.055 \\
\hline $\begin{array}{l}\text { Biopsy device } \\
\text { CNB vs VAB }\end{array}$ & 0.407 & $0.192-0.862$ & 0.018 & 0.327 & $0.119-0.828$ & 0.018 \\
\hline $\begin{array}{l}\text { Estrogen receptor } \\
\text { Negative vs Positive }\end{array}$ & 0.304 & $0.121-0.765$ & 0.009 & 0.978 & $0.195-4.904$ & 0.978 \\
\hline $\begin{array}{l}\text { Progesterone receptor } \\
\text { Negative vs Positive }\end{array}$ & 0.476 & $0.223-1.016$ & 0.053 & 0.882 & $0.258-3.153$ & 0.843 \\
\hline $\begin{array}{l}\text { HER2 } \\
\leq 2 \text { vs } 3\end{array}$ & 3.203 & $1.164-8.811$ & 0.020 & 1.047 & $0.230-4.652$ & 0.952 \\
\hline $\begin{array}{l}\text { Ki67 } \\
\leq 14 \% \text { vs > } 14 \%\end{array}$ & 2.866 & $1.199-6.852$ & 0.016 & 1.270 & $0.372-4.241$ & 0.698 \\
\hline $\begin{array}{l}\text { Grade of DCIS } \\
\text { Low, intermediate vs High }\end{array}$ & 4.508 & $1.767-11.501$ & 0.001 & 2.976 & $0.820-11.504$ & 0.097 \\
\hline $\begin{array}{l}\text { Comedonecrosis } \\
\text { Absence vs Presence }\end{array}$ & 1.808 & $0.877-3.728$ & 0.107 & & & \\
\hline $\begin{array}{l}\text { Intraductal calcification } \\
\text { Absence vs Presence }\end{array}$ & 1.918 & $0.747-4.922$ & 0.171 & & & \\
\hline $\begin{array}{l}\text { Lymphoid infiltrate } \\
\text { Negative, mild vs moderate, }\end{array}$ & 4.571 & $2.052-10.185$ & $<0.001$ & 2.419 & $0.839-7.138$ & 0.102 \\
\hline $\begin{array}{l}\text { Smoking status } \\
\text { Never smokers vs Smokers }\end{array}$ & 0.573 & $0.206-1.591$ & 0.281 & & & \\
\hline $\begin{array}{l}\text { Smoking status } \\
\text { Never or former smokers vs }\end{array}$ & - & - & 0.019 & - & - & 0.006 \\
\hline $\begin{array}{l}\text { Pack-years of smoking } \\
\leq 10 \text { vs } 10<\end{array}$ & 0.156 & $0.019-1.275$ & 0.050 & 0.329 & $0.014-3.122$ & 0.349 \\
\hline
\end{tabular}

\section{Discussion}

There are various reports about risk factors for finding IDC by postoperative pathological examination in cases diagnosed as DCIS by preoperative biopsy. A meta-analysis lists the following items as risk factors: biopsy devices, high-grade DCIS, tumor larger than 20 mm, palpability, and others [1]. There are also reports that list negative hormonal receptor [20, 21], HER2 overexpression [22, 23], and lymphoid infiltrate as risk factors [18, 19]. However, other reports do not identify these clinicopathologic features as risk factors [24]. No study reporting high Ki67 as a risk factor was found, 
but it was thought that DCIS with HER2 overexpression or high Ki67 had a potential risk of invasion because these features represented a high risk for postoperative DCIS recurrence [25-27].

In this study, the same clinicopathological factors as those previously reported were identified as risk factors for finding infiltration after surgery. However, the patients included in this study differed from the patients of previous reports in terms of clinicopathologic features. An important difference is the rate of IDC found by postoperative pathological examination. In the meta-analysis previously reported, the ratio was $25.9 \%$ (18.6$37.2 \%$ ) [1], while we found a higher rate of $39.1 \%$. We presume that this difference is due to the biopsy device. We have been using 16 -gauge CNB because of the physical burden on patients. As a result, IDC was found by postoperative pathological examination at a high-rate of $47.9 \%$ in cases diagnosed by CNB, which may have affected the overall rate of IDC found by postoperative pathological examination.

Another difference is that fewer patients had the clinicopathological features listed as risk factors. For example, the palpable tumor rate was 8.8 -fold higher in this study compared with a previous report [1]. Similarly, the overexpression rate of HER2 was $14.8 \%$ in this study and $28-65 \%$ in a previous report [28]. In addition, high-grade DCIS corresponded to $49.4 \%$ of cases in a previous report [1] but only $18.8 \%$ in this study. These differences may exist due to the low rate of breast cancer screening in Japan, which is lower than that in other countries at $40 \%$ [29]. The fact that various risk factors are lower than those reported previously may indicate that, in many cases, invasion already occurred at the time the patient presented to the hospital.

In this study, current-smokers were significantly less likely to find postoperative IDC compared to never-smokers and former-smokers. In addition, the risk of finding IDC decreased with increasing daily smoking and smoking duration, although these were not significant. This is supported by the results of a case-control study. In that study, the current status as a smoker was more important for the onset of DCIS than the exposure to tobacco, and the result was an inverse correlation [8]. However, experiments in vivo and in vitro have shown that tobacco components increase the malignancy of breast cancer cells [3-5]. In addition, we have shown in clinical samples that smoking can enhance HER2 expression in breast cancer and increase tumor-infiltrating lymphocyte density in the microenvironment surrounding the cancer [16, 30]. From these results, we speculate that smoking may affect DCIS during the beginning of the disease, causing it to acquire invasive ability at an early stage and resulting in IDC identified by biopsy at initial diagnosis. Our speculation is supported by a case-control analysis that listed smoking as a risk of developing luminal A IDC, while it was associated with reduced risk of developing luminal A DCIS; however, the associations were not significant [31].

Some of the limitations of this study are the higher rate of postoperative IDC detection and the fewer number of patients with risk factors than those previously reported. In addition, the data regarding smoking habits may not be entirely accurate because it was based on self-reporting. Because some studies listed younger age as a risk factor for finding invasion by postoperative pathological examination [32-34], the fact that the age distribution was disproportional between smoking and non-smoking patients may represent a small limitation.

\section{Conclusions}

This study suggests that tobacco usage may have an influence on the progression from DCIS to IDC, contributing to the elucidation of the underlying mechanism involved in this change.

\section{Abbreviations}

CNB

core needle biopsy, CT:computed tomography, DCIS:ductal carcinoma in situ, ER:estrogen receptor, HER2:human epidermal growth factor receptor 2, IDC:invasive ductal carcinoma, OR:odds ratio, PgR:progesterone receptor, VAB:vacuum-assisted biopsy.

\section{Declarations}

\section{Ethics approval and consent to participate}

Written informed consent was obtained from all subjects. This research conformed to the provisions of the Declaration of Helsinki in 2013 . All patients were informed of the investigational nature of this study and provided their written, informed consent. The study protocol was approved by the Ethics Committee of Osaka City University (\#926).

\section{Consent for publication}

Not applicable.

\section{Availability of data and materials}

The datasets used and/or analyzed during the current study are available from the corresponding author on reasonable request.

\section{Competing interests}

The authors declare that they have no competing interests. 


\section{Funding}

This study was supported in part by Grants-in-Aid for ScientificResearch (KAKENHI, Nos. 17K10559, 19K18067 and 20K08938) from the Ministry ofEducation, Science, Sports, Culture and Technology of Japan.

\section{Authors' contributions}

KTparticipated in the design of the study and drafted the manuscript. SK participated in the design of the study and manuscript editing. YA, WG, RK, AY, TM, MS,and TT helped with study data collection and manuscript preparation. HF helped with study data collection and participated in its design. $\mathrm{KH}$ and $\mathrm{MO}$ conceived the study, and participated in its design and coordination and helped to draft the manuscript. All authors have read and approved the final manuscript.

\section{Acknowledgements}

We thank Yayoi Matsukiyo and Tomomi Okawa (Department of Breast and Endocrine Surgery, Osaka City University Graduate School of Medicine) for helpful advice regarding data management.

\section{Author's information}

${ }^{1}$ Department of Breast and Endocrine Surgery, Osaka City University Graduate School of Medicine, 1-4-3 Asahi-machi, Abeno-ku, Osaka 545-8585, Japan.

${ }^{2}$ Department of Gastrointestinal Surgery, Osaka City University Graduate School of Medicine, 1-4-3 Asahi-machi, Abeno-ku, Osaka 545-8585, Japan.

${ }^{3}$ Department of Scientific and Linguistic Fundamentals of Nursing, Osaka City University Graduate School of Nursing, 1-5-17 Asahi-machi, Abeno-ku, Osaka 545-0051, Japan.

\section{References}

1. Brennan ME, Turner RM, Ciatto S, Marinovich ML, French JR, Macaskill P, Houssami N. Ductal carcinoma in situ at core-needle biopsy: metaanalysis of underestimation and predictors of invasive breast cancer. Radiology. 2011;260:119-28.

2. Dossus L, Boutron-Ruault MC, Kaaks R, Gram IT, Vilier A, Fervers B, Manjer J, Tjonneland A, Olsen A, Overvad K, et al. Active and passive cigarette smoking and breast cancer risk: results from the EPIC cohort. Int J Cancer. 2014;134:1871-88.

3. Forteza RM, Casalino-Matsuda SM, Falcon NS, Valencia Gattas M, Monzon ME. Hyaluronan and layilin mediate loss of airway epithelial barrier function induced by cigarette smoke by decreasing E-cadherin. J Biol Chem. 2012;287:42288-98.

4. Melendez-Colon VJ, Luch A, Seidel A, Baird WM. Cancer initiation by polycyclic aromatic hydrocarbons results from formation of stable DNA adducts rather than apurinic sites. Carcinogenesis. 1999;20:1885-91.

5. Nishioka T, Kim HS, Luo LY, Huang Y, Guo J, Chen CY. Sensitization of epithelial growth factor receptors by nicotine exposure to promote breast cancer cell growth. Breast Cancer Res. 2011;13:R113.

6. Kabat GC, Kim M, Kakani C, Tindle H, Wactawski-Wende J, Ockene JK, Luo J, Wassertheil-Smoller S, Rohan TE. Cigarette smoking in relation to risk of ductal carcinoma in situ of the breast in a cohort of postmenopausal women. Am J Epidemiol. 2010;172:591-9.

7. Claus EB, Stowe M, Carter D. Breast carcinoma in situ: risk factors and screening patterns. J Natl Cancer Inst. 2001;93:1811-7.

8. Trentham-Dietz A, Nichols HB, Egan KM, Titus-Ernstoff L, Hampton JM, Newcomb PA. Cigarette smoking and risk of breast carcinoma in situ. Epidemiology. 2007;18:629-38.

9. Kuerer HM, Albarracin CT, Yang WT, Cardiff RD, Brewster AM, Symmans WF, Hylton NM, Middleton LP, Krishnamurthy S, Perkins GH, et al. Ductal carcinoma in situ: state of the science and roadmap to advance the field. J Clin Oncol. 2009;27:279-88.

10. Erbas B, Provenzano E, Armes J, Gertig D. The natural history of ductal carcinoma in situ of the breast: a review. Breast Cancer Res Treat. 2006;97:135-44.

11. Dupont WD, Parl FF, Hartmann WH, Brinton LA, Winfield AC, Worrell JA, Schuyler PA, Plummer WD. Breast cancer risk associated with proliferative breast disease and atypical hyperplasia. Cancer. 1993;71:1258-65.

12. Carter CL, Corle DK, Micozzi MS, Schatzkin A, Taylor PR. A prospective study of the development of breast cancer in 16,692 women with benign breast disease. Am J Epidemiol. 1988;128:467-77.

13. Lakhani SR, Chaggar R, Davies S, Jones C, Collins N, Odel C, Stratton MR, O'Hare MJ. Genetic alterations in 'normal' luminal and myoepithelial cells of the breast. J Pathol. 1999;189:496-503.

14. Ambrosone CB, Kropp S, Yang J, Yao S, Shields PG, Chang-Claude J. Cigarette smoking, N-acetyltransferase 2 genotypes, and breast cancer risk: pooled analysis and meta-analysis. Cancer Epidemiol Biomarkers Prev. 2008;17:15-26.

Page 13/14 
15. Hecht SS. Tobacco smoke carcinogens and breast cancer. Environ Mol Mutagen. 2002;39:119-26.

16. Takada K, Kashiwagi S, Asano Y, Goto W, Takahashi K, Fujita H, Takashima T, Tomita S, Hirakawa K, Ohira M. Clinical verification of the relationship between smoking and the immune microenvironment of breast cancer. J Transl Med. 2019;17:13.

17. Allred DC, Mohsin SK, Fuqua SA. Histological and biological evolution of human premalignant breast disease. Endocr Relat Cancer. 2001;8:4761.

18. Hoorntje LE, Schipper ME, Peeters PH, Bellot F, Storm RK, Borel Rinkes IH. The finding of invasive cancer after a preoperative diagnosis of ductal carcinoma-in-situ: causes of ductal carcinoma-in-situ underestimates with stereotactic 14-gauge needle biopsy. Ann Surg Oncol. 2003;10:74853.

19. Go EM, Chan SK, Vong JS, Lui PC, Chan AW, Ma TK, Ang MA, Law BK, Tan PH, Tse GM. Predictors of invasion in needle core biopsies of the breast with ductal carcinoma in situ. Mod Pathol. 2010;23:737-42.

20. Ozkan-Gurdal S, Cabioglu N, Ozcinar B, Muslumanoglu M, Ozmen V, Kecer M, Yavuz E, Igci A. Factors predicting microinvasion in Ductal Carcinoma in situ. Asian Pac J Cancer Prev. 2014;15:55-60.

21. Lee SK, Yang JH, Woo SY, Lee JE, Nam SJ. Nomogram for predicting invasion in patients with a preoperative diagnosis of ductal carcinoma in situ of the breast. Br J Surg. 2013;100:1756-63.

22. Roses RE, Paulson EC, Sharma A, Schueller JE, Nisenbaum H, Weinstein S, Fox KR, Zhang PJ, Czerniecki BJ. HER-2/neu overexpression as a predictor for the transition from in situ to invasive breast cancer. Cancer Epidemiol Biomarkers Prev. 2009;18:1386-9.

23. Kondo T, Hayashi N, Ohde S, Suzuki K, Yoshida A, Yagata H, Niikura N, Iwamoto T, Kida K, Murai M, et al. A model to predict upstaging to invasive carcinoma in patients preoperatively diagnosed with ductal carcinoma in situ of the breast. J Surg Oncol. 2015;112:476-80.

24. Meurs CJC, van Rosmalen J, Menke-Pluijmers MBE, Ter Braak BPM, de Munck L, Siesling S, Westenend PJ. A prediction model for underestimation of invasive breast cancer after a biopsy diagnosis of ductal carcinoma in situ: based on 2892 biopsies and 589 invasive cancers. Br J Cancer. 2018;119:1155-62.

25. Si J, Yang B, Guo R, Huang N, Quan C, Ma L, Xiu B, Cao Y, Tang Y, Shen L, et al. Factors associated with upstaging in patients preoperatively diagnosed with ductal carcinoma in situ by core needle biopsy. Cancer Biol Med. 2019;16:312-8.

26. Rakovitch E, Nofech-Mozes S, Hanna W, Narod S, Thiruchelvam D, Saskin R, Spayne J, Taylor C, Paszat L. HER2/neu and Ki-67 expression predict non-invasive recurrence following breast-conserving therapy for ductal carcinoma in situ. Br J Cancer. 2012;106:1160-5.

27. Davis JE, Nemesure B, Mehmood S, Nayi V, Burke S, Brzostek SR, Singh M. Her2 and Ki67 Biomarkers Predict Recurrence of Ductal Carcinoma in Situ. Appl Immunohistochem Mol Morphol. 2016;24:20-5.

28. Siziopikou KP, Anderson SJ, Cobleigh MA, Julian TB, Arthur DW, Zheng P, Mamounas EP, Pajon ER, Behrens RJ, Eakle JF, et al. Preliminary results of centralized HER2 testing in ductal carcinoma in situ (DCIS): NSABP B-43. Breast Cancer Res Treat. 2013;142:415-21.

29. Uchida K, Ohashi H, Kinoshita S, Nogi H, Kato K, Toriumi Y, Yamashita A, Kamio M, Mimoto R, Takeyama H. Breast cancer screening and the changing population pyramid of Japan. Breast Cancer. 2015;22:172-6.

30. Takada K, Kashiwagi S, Asano Y, Goto W, Kouhashi R, Yabumoto A, Morisaki T, Fujita H, Shibutani M, Takashima T, et al. The effect of smoking on biological change of recurrent breast cancer. J Transl Med. 2020;18:153.

31. Tsai YF, Tseng LM, Lien PJ, Hsu CY, Lin YS, King KL, Wang YL, Chao TC, Liu CY, Chiu JH, Yang MH. HER2 immunohistochemical scores provide prognostic information for patients with HER2-type invasive breast cancer. Histopathology. 2019;74:578-86.

32. Marques LC, Marta GN, de Andrade JZ, Andrade D, de Barros A, Andrade FEM. Is it possible to predict underestimation in ductal carcinoma in situ of the breast? Yes, using a simple score! Eur J Surg Oncol. 2019;45:1152-5.

33. Yen TW, Hunt KK, Ross MI, Mirza NQ, Babiera GV, Meric-Bernstam F, Singletary SE, Symmans WF, Giordano SH, Feig BW, et al. Predictors of invasive breast cancer in patients with an initial diagnosis of ductal carcinoma in situ: a guide to selective use of sentinel lymph node biopsy in management of ductal carcinoma in situ. J Am Coll Surg. 2005;200:516-26.

34. Doebar SC, de Monye C, Stoop H, Rothbarth J, Willemsen SP, van Deurzen CH. Ductal carcinoma in situ diagnosed by breast needle biopsy: Predictors of invasion in the excision specimen. Breast. 2016;27:15-21.

\section{Supplementary Files}

This is a list of supplementary files associated with this preprint. Click to download.

- Additionalfile1TableS1.docx

- Additionalfile2Tables2.docx 